\title{
PERFIL SOCIODEMOGRÁFICO DOS AGENTES COMUNITÁRIOS DE SAÚDE DA ESTRATÉGIA SAÚDE DA FAMÍLIA NO MUNICÍPIO DE PALMAS-TO
}

Sociodemographic profile of the community health agents of the strategy 'family health' in the city of Palms-To

\section{Perfil Sociodemográfico De Los Agentes Comunitarios De Salud De La Estrategia} 'Salud De La Familiar' En La Ciudad De Palmas-To

\author{
Maria Sortênia Alves Guimarães ${ }^{1 *}$, Maria Fátima de Sousa ${ }^{2}$, Talita Buttarello Mucari ${ }^{1}$ \\ ${ }^{1}$ Docente do Curso de Medicina, Universidade Federal do Tocantins, Campus de Palmas, Tocantins, Brasil. \\ ${ }^{2}$ Docente do Departamento de Saúde Coletiva, da Faculdade de Ciências da Saúde, Universidade de Brasília, \\ Distrito Federal, Brasil.
}

*Correspondência: Coordenação do Curso de Medicina - Universidade Federal do Tocantins - Av. NS 15, 109 Norte, Palmas, Tocantins, Brasil.CEP:77.010-090.e-mail: msortenia@hotmail.com

\section{Artigo recebido em 09/03/2017 aprovado em 19/07/2017 publicado em 08/08/2017.}

\section{RESUMO}

O Agente Comunitário de Saúde, nas duas últimas décadas, vem representando, no setor saúde, um profissional estratégico para aproximar as famílias e comunidades dos processos de trabalho em saúde. Entra em cena um novo ator político para contribuir no fortalecimento dos Sistemas Municipais de Saúde. Este artigo tem como objetivo traçar o perfil sociodemográfico desse profissional no Município de Palmas-TO. Trata-se de estudo tipo survey exploratório, realizada com 246 Agentes Comunitários de Saúde, entre janeiro e abril de 2014. A coleta de dados foi por questionários, em observância a Resolução nº66/2012 do Conselho Nacional de Saúde. Verificou-se que a maioria dos Agentes Comunitário de Saúde pertence ao gênero feminino (85,89\%), está entre 30 e 44 anos de idade $(68,57 \%)$ e apresenta ensino médio completo $(54,06 \%)$. Dos 246 entrevistados, 38,21\% fizeram cursos técnicos. Dentre esses 62,77\% em Técnico de Agente Comunitário de Saúde. A maioria (55,19\%) está na profissão entre 10 e 14,9 anos. O número de visitas domiciliares realizadas pelos Agentes Comunitário de Saúde foi em média 9,32 visitas/dia. Evidencia-se no estudo que o perfil que emerge da pesquisa é semelhante aqueles desenhado pela Política Nacional da Atenção Básica, ainda assim, destaca-se a necessidade de inserção dos mesmos em projetos de educação permanente.

Palavras-chave: Agente Comunitário de Saúde; Estratégia Saúde da Família; Comunidade.

\begin{abstract}
In the last two decades, the Community Health Agent has represented, in the health sector, a strategic professional that brings together families and communities in the work processes of the health field. A new political actor comes on stage to contribute to the strengthening of the Municipal Health Systems. This article aims to outline the demographic profile of this professional in the city of Palmas-TO. This is an exploratory study survey that was conducted with 246 Community Health Agents between January and April 2014. The data collection was made via questionnaires, observing the Resolution 466/2012 of the National Health Council. We found that most of the Community Health Agents are females (85.89\%) and they are between 30 and 44 years old (68.57\%) and have completed secondary education (54.06\%). Of the 246 respondents, 38.21\% had technical courses. Of these, 62.77\% are Technical Community Health Agents. Most of them (55.19\%) are in the profession between 10 and 14.9 years. The number of home visits by the Community Health Agents is approximately 9.32 visits per day. It is evident in the study that the profile that emerges from the research is like those designed by the National Primary Care Policy, nonetheless, it is highlighted the need for inclusion of them in continuing education projects.
\end{abstract}


Keywords: Community Health Agent; Family Health Strategy; Community.

\section{RESUMEN}

Enlas últimas dos décadas, el Agente Comunitario de Salud ha representado enel sector de lasaludun enfoque estratégico para lasfamilias y las comunidades profesionalesenlosprocesos de trabajoensalud. Aparece enescenaunnuevoactor político de contribuir al fortalecimiento de los Sistemas Municipales de Salud. Este artículo tiene como objetivo trazarel perfil demográfico delprofesionalenlaciudad de Palmas-TO. Este es unestudio tipo encuestaexploratoria. El fue realizado con 246 trabajadorescomunitarios de salud entre enero y abril de 2014. La recolección de datosfue a través de cuestionarios de acuerdoconlaobservación de laResolución 466/2012 delConsejo Nacional de Salud. Se encontró que lamayoría de los Agentes Comunitarios de Saludsonmujeres (85,89\%) y tienen entre 30 y 44 años (68,57\%). Tambiénhan completado laeducación secundaria (54.06\%). De los 246 encuestados, $38.21 \%$ tenían cursos técnicos. De estos, 62,77\% tenían curso técnico de Agente Comunitario de Salud. Una mayoría de (55,19\%) se encuentraenlaprofesión entre 10 y 14,9 años. El número mediano de visitas a domicilio de los Agentes Comunitarios de Saludfue 9,32 visitas por día. Es evidente enelestudio que el perfil que se desprende de lainvestigación es como losdiseñados por la Política Nacional de Atención Primaria, sin embargo, se sobresalelanecesidad de lainclusión de este tipo de proyectoseneducación continua.

Palabras clave: Agente Comunitario de Salud; EstrategiaSalud de laFamilia; Comunidad.

\section{INTRODUÇÃO}

O Programa Saúde da Família (PSF) surgiu em 1994, sob a égide da discussão da reformulação do sistema de saúde, a qual ampliou a concepção de atenção, definida no Decreto $\mathrm{N}^{\circ}$. 7.508/2011. O PSF teve como precursor o Programa de Agentes Comunitários de Saúde (PACS), implantado em 1991, e adotou, como referência, as experiências internacionais e nacionais de extensão da cobertura à saúde por meio de assistência às famílias no próprio domicílio em articulação com as unidades de saúde (BRASIL, 2000 a). Assim em 1994, surge no Brasil o Programa de Saúde da Família, hoje denominado de Estratégia de Saúde da Família (ESF) ${ }^{1}$, que se trata de uma política de saúde implementada na maioria dos municípios brasileiros, que prioriza dentre outras ações, a promoção, proteção e recuperação da saúde dos indivíduos e da família de forma integral e contínua (CARVALHO, 2009).

\footnotetext{
${ }^{1}$ Atualmente, o PSF é definido como Estratégia de Saúde da Família, ao invés de programa, visto que o termo programa aponta para uma atividade com início, desenvolvimento e finalização.
}

A estratégia deve oferecer, em um território definido, atenção integral e contínua à saúde dos indivíduos e da comunidade, com ações de promoção, proteção e recuperação da saúde. As ações desenvolvidas devem estar centradas na família, percebida a partir do seu ambiente socioeconômico e cultural (OLIVEIRA JÚNIOR, 2012).

Uma diretriz do Ministério da Saúde (MS) é que o trabalho seja estruturado em uma Unidade de Saúde da Família (USF), onde fica sediada a equipe multiprofissional com responsabilidade por uma determinada população a ela vinculada (BRASIL, 2000 b).

Esta organização favorece o estabelecimento de vínculos de responsabilidade e confiança entre profissionais e famílias, permitindo uma compreensão ampliada do processo saúde-doença e da necessidade de intervenções a partir dos problemas e demandas identificadas (BRASIL, 2005).

O trabalho dos profissionais de saúde na ESF é voltado para a assistência integral e contínua de todos os membros da família, vinculados à Unidade Básica de Saúde (UBS), em cada fase de seu ciclo de 
vida, sem perder de vista seu contexto familiar e social (NEGRI et al., 2004; ROSA ; LABATE, 2005). De acordo com o Ministério da Saúde, as equipes podem identificar fatores de riscos a que a população está exposta e resolver $85 \%$ dos problemas de saúde em sua comunidade, prestando um bom atendimento, prevenindo doenças, evitando internações e melhorando a qualidade de vida da população (BRASIL, 2000 c).

O Agente Comunitário de Saúde (ACS) é um importante agente social, que funciona como elo entre a equipe e a comunidade; foi introduzido nos municípios brasileiros a partir do início dos anos 1990. Em 2002, a Lei Federal 10.507 regulamentou a profissão e estabeleceu critérios para contratação do ACS, que deve ser um morador da comunidade e que irá desenvolver atividades de prevenção de doenças e promoção da saúde, por meio de ações domiciliares ou comunitárias, além da necessidade de preencher os seguintes requisitos para o exercício da profissão: haver concluído o ensino fundamental; haver concluído com aproveitamento curso de qualificação básica para a formação de Agente Comunitário de Saúde (BRASIL, 2002).

A Portaria n 2.488 , de 21 de outubro de 2011 estabelece a revisão de diretrizes e normas para a organização da Atenção Básica para a ESF e o PACS, assim como define as atribuições de cada categoria profissional existente na equipe de saúde da família. Os ACS têm entre suas funções: cadastrar todas as pessoas de sua micro-área e manter os cadastros atualizados; desenvolver ações de integração entre a equipe de saúde e a população adscrita à Unidade Básica de Saúde; desenvolver atividades de promoção da saúde, de prevenção das doenças e de agravos e de vigilância à saúde, através de ações educativas individuais e coletivas na comunidade (BRASIL, 2011).
O número de ACS não pode exceder o limite de doze por equipe e deve ser suficiente para atender toda população cadastrada, sendo cada um responsável pelo acompanhamento de no máximo 750 pessoas (BRASIL, 2006).

Para que o Sistema Único de Saúde (SUS) se torne mais forte e estabelecido perante a sociedade, o ACS tem tido um papel importante, pois promove a integração dos serviços de saúde da Atenção Primária à Saúde e as pessoas da comunidade (BRASIL, 2009).

O ACS é percebido, desta forma, como o elo entre a equipe de saúde e a comunidade, sendo o principal responsável pela efetivação do vínculo nessa relação (SEOANE, 2009). Presta apoio à população diretamente nas residências, identificando questões não somente de saúde, mas tantas outras que podem receber suporte da unidade de saúde. Orienta, conduz e acompanha processos que podem ser importantes para a população do bairro que atende (MALFITANO, 2009). É um promotor da saúde, por excelência, um educador permanente, na defesa da vida (SOUSA, 2001).

Diante disso, o ACS pode ser visto como um trabalhador sui generis, uma vez que suas funções transcendem o campo da saúde, exigindo-lhe atenção a múltiplos aspectos das condições de vida da população (GOMES et al., 2009).

Considerando que o Tocantins é o mais novo Estado brasileiro, onde poucos estudos com ACS foram realizados, este trabalho torna-se de suma relevância para a saúde pública tocantinense. Assim, a pesquisa realizada objetivou traçar o perfil dos ACS do Município de Palmas - Tocantins - Brasil.

\section{MATERIAIS E MÉTODOS}


Este trabalho foi um estudo do tipo survey exploratório. $\mathrm{O}$ estudo foi realizado na cidade de Palmas, Estado do Tocantins, pertencente à região norte do Brasil, que localiza-se à longitude de $48^{\circ} 21^{\prime}$ $37^{\prime \prime} \mathrm{W}$ e latitude de $10^{\circ} 12^{\prime} 46^{\prime \prime} \mathrm{S}$. O município de Palmas está localizado na região central do Estado do Tocantins. A área de 2.218,94 $\mathrm{km}^{2}$ representa $0,79 \%$ do território estadual e possui como limite Norte os municípios de Aparecida do Rio Negro e Lajeado, ao Sul Monte do Carmo e Porto Nacional, ao Leste Santa Tereza do Tocantins e Novo Acordo, e a Oeste Miracema do Tocantins (Figura 1).

Figura 1. Divisão político-administrativa do estado e limites municipais de Palmas - TO, Brasil, 2014.
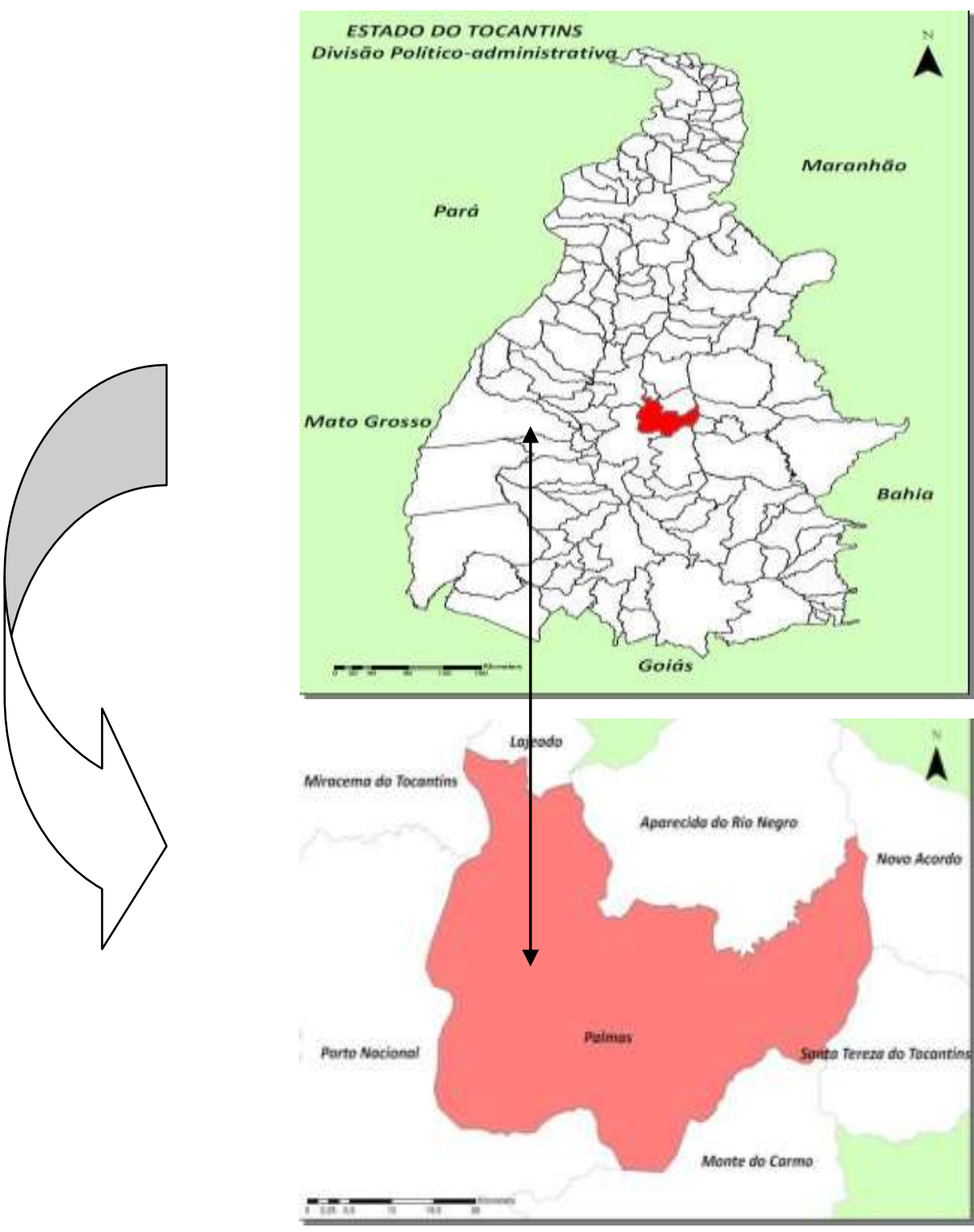

Fonte: Secretaria Municipal De Assuntos Jurídicos - Prefeitura Municipal De Palmas, 2014.

A cidade foi planejada para ser a capital, criada em 20 de maio de 1989 (pouco tempo depois da criação do Estado) e instalada em $1^{\circ}$ de janeiro de 1990. Possuía população de 228.332 habitantes, segundo o censo do Instituto Brasileiro de Geografia e Estatística de 2010 (IBGE, 2010), com densidade demográfica de 102,90 habitantes por $\mathrm{km}^{2}$. No entanto, no Diário Oficial da União de 28 de agosto 
de 2014, o IBGE divulgou dados estimativos em que Palmas sendo a capital brasileira que apresentou o maior crescimento geométrico entre 2013 e 2014 (2,91\%), estimando-se uma população de 265.409 habitantes em 2014, conforme nota técnica do IBGE (2014). O bioma principal da cidade é o Cerrado; e o clima é quente e seco, com médias de temperatura em torno de $26,5^{\circ} \mathrm{C}$ (INMET, 2014).

Os sujeitos da pesquisa foram os ACS que atuam nas USF do Município de Palmas - TO. Do total de 408 ACS, atualmente 20 não estão em exercício, sendo: 14 afastados por desvio de função, três de licença médica, um com Mandato Classista (Representante do Conselho Tutelar) e dois com redução de carga horária (junta médica). Foram considerados para o cálculo da amostra do estudo, apenas os 388 ACS em exercício da função nas unidades de saúde estudadas. A amostra selecionada foi de 246 indivíduos, que responderam os instrumentos de coleta de dados (Tabela 1), estatisticamente tal amostra representa uma margem de erro de aproximadamente 4\%. Foi realizada amostragem aleatória e estratificada, de modo que os ACS de todas as unidades de saúde da família participassem da pesquisa. A adesão da participação dos ACS no estudo foi de livre e espontânea vontade, respeitando os preceitos éticos da pesquisa, conforme reza as diretrizes e normas regulamentadoras estabelecidas pela Resolução no 466/ 2012 do Conselho Nacional de Saúde. O estudo apresentou questões que não totalizaram 246 respostas, porque alguns ACS, não responderam na totalidade o questionário. Assim, o cálculo foi realizado sobre o número de questões respondidas.

Tabela 1. Distribuição dos Agentes Comunitários de Saúde (ACS) em exercício e entrevistados, por Unidade e Região de Palmas - TO, Brasil, 2014.

\begin{tabular}{|c|c|c|}
\hline $\begin{array}{c}\text { Unidades de Saúde da } \\
\text { Família - USF }\end{array}$ & $\begin{array}{c}\text { Número de ACS em } \\
\text { exercício }\end{array}$ & $\begin{array}{c}\text { Número de ACS } \\
\text { entrevistados }\end{array}$ \\
\hline \multicolumn{3}{|l|}{ Região Norte } \\
\hline Policlínica 303 Norte & 12 & 06 \\
\hline 307 Norte & 11 & 08 \\
\hline 403 Norte & 12 & 06 \\
\hline 405 Norte & 18 & 15 \\
\hline 406 Norte & 08 & 03 \\
\hline 503 Norte & 13 & 07 \\
\hline 508 Norte & 06 & 04 \\
\hline 603 Norte & 08 & 05 \\
\hline Total da Região Norte & 88 & 54 \\
\hline \multicolumn{3}{|l|}{ Região Central } \\
\hline Policlínica 108 Sul & 17 & 12 \\
\hline Loiane Moreno Vieira & 13 & 08 \\
\hline $403 \mathrm{Sul}$ & 17 & 11 \\
\hline $712 \mathrm{Sul}$ & 19 & 08 \\
\hline 806 Sul & 16 & 08 \\
\hline $1004 \mathrm{Sul}$ & 13 & 08 \\
\hline $1103 \mathrm{Sul}$ & 11 & 09 \\
\hline $1106 \mathrm{Sul}$ & 06 & 03 \\
\hline Valéria Martins Pereira & 13 & 10 \\
\hline Total da Região Central & 125 & 77 \\
\hline
\end{tabular}




\begin{tabular}{ccc}
\hline Eugênio P. da Silva & 17 & 09 \\
Aureny II & 12 & 07 \\
Liberdade & 06 & 03 \\
José Lúcio de Carvalho & 16 & 08 \\
Laurides Lima Milhomem & 21 & 17 \\
Novo Horizonte & 09 & 06 \\
Alto Bonito & 04 & 04 \\
Santa Bárbara & 13 & 07 \\
Taquari & 11 & 06 \\
Bela Vista & 06 & 04 \\
Setor Sul & 08 & 06 \\
Santa Fé & 15 & 09 \\
Mora do Sol & 10 & 09 \\
Taquaruçu & 07 & 04 \\
Total da Região Sul & $\mathbf{1 5 5}$ & $\mathbf{9 9}$
\end{tabular}

\begin{tabular}{cc}
\hline Buritirana & 07 \\
Taquaruçu Grande & 13 \\
Total da Região Rural & $\mathbf{2 0}$
\end{tabular}

$07 \quad 07$

$13-09$

2016

TOTAL GERAL

Esta pesquisa foi submetida ao Comitê de Ética da Secretaria Municipal de Saúde do Município de Palmas - TO, em Agosto de 2013, com a aprovação deste comitê em Setembro de 2013. Posteriormente, em 01 de Outubro de 2013, foi submetido à análise do Comitê de Ética em Pesquisa da Universidade Federal do Tocantins, com aprovação no mês de Dezembro de 2013. O mesmo encontra-se cadastrado na Plataforma Brasil, sob o número 24966514.8.0000.5519.

Neste estudo, buscando atingir os objetivos, foi elaborado questionário, objetivando traçar o perfil da população do estudo. A coleta dos dados foi realizada no período de janeiro à abril de 2014.

O banco de dados após coleta foi construído no programa EPI-INFO (versão 7). A tabulação dos dados e análise estatística foram realizadas pelo mesmo software. Foram construídos tabelas de distribuição de freqüências, além de calculadas medidas descritivas que facilitam a interpretação dos dados, bem como intervalos de confiança de $95 \%$ para tais frequências.

\section{RESULTADOS E DISCUSSÃO}

O perfil do profissional estudado, o ACS, pode ser observado na Tabela 2, que mostra a sua caracterização demográfica e social e sua distribuição em frequências absolutas e percentuais. 
Tabela 2. Distribuição de frequiências de gênero, escolaridade, faixa etária e tempo de serviço dos Agentes Comunitários de Saúde, Palmas - TO, Brasil, 2014

\begin{tabular}{|c|c|c|c|}
\hline Variáveis & $\mathbf{N}$ & $\%$ & $\begin{array}{c}\text { Intervalo de Confiança } \\
(\mathbf{9 5 \%})\end{array}$ \\
\hline \multicolumn{4}{|l|}{ GÊNERO } \\
\hline Feminino & 207 & 85,89 & $80,85-90,03$ \\
\hline Masculino & 34 & 14,11 & $9,97-19,15$ \\
\hline Total & 241 & 100,00 & \\
\hline \multicolumn{4}{|l|}{ ESCOLARIDADE } \\
\hline EFI & 1 & 0,41 & $0,01-2,24$ \\
\hline EFC & 4 & 1,63 & $0,44-4,11$ \\
\hline EMI & 3 & 1,22 & $0,25-3,25$ \\
\hline EMC & 133 & 54,06 & $50,29-57,83$ \\
\hline ESI & 49 & 19,92 & $16,90-22,94$ \\
\hline ESC & 51 & 20,73 & $17,67-23,79$ \\
\hline Pós-Graduação & 5 & 2,03 & $0,66-4,68$ \\
\hline Total & 246 & 100,00 & \\
\hline \multicolumn{4}{|l|}{ FAIXA ETÁRIA } \\
\hline 25 a 29 anos & 11 & 4,49 & $2,26-7,89$ \\
\hline 30 a 34 anos & 53 & 21,63 & $16,64-27,32$ \\
\hline 35 a 39 anos & 61 & 24,90 & $19,61-30,80$ \\
\hline 40 a 44 anos & 54 & 22,04 & $17,01-27,76$ \\
\hline 45 a 49 anos & 38 & 15,51 & $11,22-20,66$ \\
\hline Acima de 50 anos & 28 & 11,43 & $7,73-16,09$ \\
\hline Total & 245 & 100,00 & \\
\hline
\end{tabular}

\begin{tabular}{ll}
\hline TEMPO DE \\
SERVIÇO
\end{tabular}

Até 4,9 anos

$2 \quad 0,83$

$0,12-1,53$

De 5 a 9,9 anos

$81 \quad 33,61$

$29,96-37,26$

De 10 a 14,9 anos

$133 \quad 55,19$

$51,35-59,03$

A partir de 15 anos

$25 \quad 10,37$

$8,02-12,72$

Total

$241 \quad 100,00$

$\mathrm{n}=$ freqüência absoluta; \% = porcentagem; EFI= Ensino Fundamental Completo; EFC= Ensino Fundamental Incompleto; EMI = Ensino Médio Completo; EMC = Ensino Médio Incompleto; ESI = Ensino Superior Incompleto; EMI = Ensino Superior Completo.

A Tabela 2 revela que nas Unidades de Saúde da Família de Palmas - TO, existe predominância de ACS do sexo feminino $(85,89 \%)$. Em relação à "tendência" pelo gênero feminino, esta perece seguir desde os primórdios do Programa ao ser implantado no Ceará em 1987 (SILVA ; DALMASO, 2002).
Lunardelo (2004) recorre à gênese da Estratégia Saúde da Família (ESF) para demonstrar que, desde a implantação do PACS, a contratação de mulheres foi priorizada, com a intenção de causar impacto na condição social dessa parcela da população por meio do trabalho remunerado. 
Essa preferência baseou-se no fato de que as mulheres transformariam a sua condição social, através do trabalho remunerado, apesar de receberem apenas o salário mínimo e, com isso, incentivariam um posicionamento mais ativo de outras mulheres da comunidade onde moravam. Outro fato importante que levou a escolha das mulheres para exercer essa atividade era a possibilidade de promover a educação em saúde com o grupo materno-infantil, na tentativa de reduzir a morbidade e mortalidade dessa população, por meio de ações que aumentavam o índice de aleitamento materno e prevenção da desidratação, proveniente da diarréia (MINAYO, 1990).

Diante do exposto, constata-se que o programa originou-se de emprego de mulheres pobres e não capacitadas, devendo cumprir uma ação de urgência, na qual se acreditava conseguir resultados com ações simples. De 1987 até hoje, o ACS se tornou profissão e continua sendo, assim como muitas outras, uma profissão feminina (ÁVILA, 2006).

Esse fato pode estar intensamente ligado ao papel de cuidadora que a mulher exerce na sociedade, sendo as principais responsáveis pela educação e pela alimentação das crianças e pelos cuidados proporcionados aos idosos da família (ELLIS; HARTLEY, 1998).

Esta expectativa mantém-se forte na estrutura do programa atual, embora a participação de homens traga novos olhares e novos encontros, repercutindo de forma positiva na promoção da saúde das famílias acompanhadas pelas equipes.

Investigações desenvolvidas com ACS em outros municípios brasileiros demonstraram dados semelhantes a este, isto é, a predominância do trabalho feminino (MARTINS et al., 1996; ANDRADE, 1998; SILVA ; DALMASO, 2002; FERRAZ ; AERTS, 2005; SANTOS, 2006;
AZAMBUJA et al. 2007, MARQUES, 2008). Observa-se que estes estudos foram realizados em períodos diferentes e que não houveram mudanças neste aspecto ao longo dos anos.

Segundo os dados do estudo, também foi possível observar, ainda na Tabela 2 , que as faixas etárias que mais concentraram agentes foram entre 30 e 44 anos de idade $(68,57 \%)$, isto é, adultos jovens. Um dos pré-requisitos do Ministério da Saúde é que tenham idade acima de 18 anos, não sendo estabelecido um limite máximo (BRASIL, 2001).

Este perfil etário mostra uma força de trabalho em plena maturidade produtiva e uma população economicamente ativa de adultos jovens. Em outros estudos (ANDRADE, 1998; SILVA; DALMASO, 2002; TEIXEIRA ; MISHIMA, 2000; SANTOS, 2011), os autores também encontraram uma concentração de ACS entre 30 a 49 anos. Silva e Dalmaso (2002) destacaram que, para muitos, ser agente comunitário de saúde foi uma oportunidade de ingresso e reingresso no mercado de trabalho.

Com relação à escolaridade dos entrevistados, é observado um nível de escolaridade contemplador ou superior ao exigido pelo Ministério da Saúde, pois $54,06 \%$ (133) participantes concluíram o ensino médio, 19,92 \% (51) concluíram o ensino superior e $2,03 \%$ (5) informaram ser pósgraduados.

Dos 246 entrevistados, 38,21\% fizeram cursos técnicos. Dentre esses $62,77 \%$ em Técnico de Agente Comunitário de Saúde.

No Município de Palmas, o Curso Técnico de Agente Comunitário de Saúde foi ofertado uma única vez, no período de 2005 a 2008, e não há previsão de oferta do curso para novas turmas (informação verbal) ${ }^{2}$

\footnotetext{
${ }^{2}$ Informação fornecida pela Escola Tocantinense do Sistema Único de Saúde (ETSUS), Palmas - TO, em agosto de 2014.
} 
A respeito dessa perspectiva formativa, o Curso Técnico de Agente Comunitário de Saúde traz reflexões sobre o fato de que o ACS tem se constituído como protagonista na luta pela melhoria das condições de vida da comunidade; sua práxis transformadora tem contribuído de forma significativa por meio de elementos educativopreventivos e de promoção da saúde no campo da atenção primária, na vigilância ambiental, sanitária e epidemiológica (MODESTO et al., 2012).

De acordo com o Ministério da Saúde, o Curso Técnico de Agente Comunitário de Saúde tem como propósito preparar os profissionais para atuar como técnicos de nível médio junto às equipes multiprofissionais que desenvolvem ações de cuidado e proteção à saúde de indivíduos e grupos sociais em domicílios e coletividades (BRASIL, 2004). Pode-se dizer que o grande ganho desse processo de formação foi a efetivação da identidade profissional dos ACS envolvidos. Antes do curso, eles não se sentiam como membros da equipe. Ocupavam-se com muitas atividades, atendendo às necessidades que surgiam, sem muita clareza do seu papel. Suas atribuições eram pouco conhecidas por eles próprios, pela equipe de saúde e pela comunidade. A partir do momento em que sua atuação foi reconhecida, aumentou a satisfação dos ACS em pertencer a uma equipe de saúde e fazer parte de um sistema de saúde estruturado nos âmbitos estadual e nacional. As vivências e os conteúdos trabalhados durante o curso trouxeram, além de uma habilitação técnica, um reconhecimento pessoal de suas potencialidades, refletindo-se na sua vida pessoal e profissional

Faz-se necessário a reflexão sobre o grau de escolaridade dos ACS, pois após a implantação do PSF, o papel do ACS foi ampliado, saindo do foco materno-infantil para a família e a comunidade, além das exigências de novas competências no campo político e social. Assim, a elevação da escolaridade dos ACS passaria a ser importante para desempenhar esse novo papel, bem mais complexo e abrangente (TOMAZ, 2002).

Entretanto, diferentes conceitos e opiniões são formados em relação à escolarização dos ACS, uma das concepções é que a sua qualificação tenderá a afastá-lo da sua principal atribuição, o de ser um trabalhador que, por vivenciar e compreender a cultura local tem a possibilidade de traduzir melhor os anseios da população com a qual convive, dessa forma, não caberia definir o seu perfil ocupacional, pois o seu trabalho está intimamente ligado à sua inserção social (LOPES et al., 2011).

Todavia, ao longo dos últimos 20 anos, a qualificação na formação dos ACS tem sido um dos objetivos da Confederação Nacional dos Agentes Comunitários de Saúde (CONACS) e, pelo menos no âmbito legal, já houve a aprovação do aumento da escolaridade e a qualificação em nível técnico, com a elaboração do "Referencial Curricular para Curso Técnico de Agente Comunitário de Saúde" no ano de 2004 (BRASIL, 2004). No entanto, esse direito conquistado, por meio de lutas e mobilizações coletivas dos ACS, não tem sido garantido, permanecendo a precarização da formação profissional.

O "Referencial Curricular para Curso Técnico de Agente Comunitário de Saúde" compreende três etapas formativas, mas até o momento somente a primeira etapa foi pactuada pelos gestores do SUS, persistindo assim uma formação apressada e fragmentada, voltada para a capacitação em serviço, e que geralmente não está de acordo com o importante papel desempenhado pelo ACS na prática da integralidade do cuidado em saúde (CHINELLIF et al. ,1990).

Quanto ao tempo de atuação na ESF, observa-se que a maioria dos entrevistados $(55,19 \%)$ está trabalhando como ACS de 10 a 14,9 anos, 
$33,61 \%$ apresentam entre 5 a 9,9 anos de serviço e 10,37\% a partir de 15 anos. Verificou-se ainda, uma baixa rotatividade desses trabalhadores, sendo que apenas $0,83 \%$ estão há menos tempo na atividade (até 4,9 anos).

Os ACS possuem baixa rotatividade na ESF e quanto maior o seu tempo de atuação maior será a sua contribuição para a equipe de saúde da família, pois o mesmo passa a conhecer melhor a comunidade e suas necessidades de saúde (FERRAZ; AERTS, 2005; MOTA ; DAVID, 2010).

Ressalta-se ainda, que a maior permanência de atuação do ACS, contribui para um melhor entendimento do seu papel, como também na construção das suas práticas cotidianas, embasadas no conhecimento da necessidade do local, além de favorecer no fortalecimento do vínculo com a comunidade.

No que tange ao número de visitas domiciliares realizadas pelos ACS no Município de Palmas, em média os ACS realizam 9,32 visitas/dia, contabilizando semanalmente em média 46,6 \pm 17,52 visitas por semana, apesar de média adequada, existe grande variação quanto ao número de visitas, demonstrada pelo alto desvio padrão, não há homogeneidade para tal variável $(\mathrm{CV}=37,6 \%)$.

No município foi pactuado com os ACS que fizessem de oito a dez visitas domiciliares por dia, o que totaliza 40 ou 50 por semana, que vem em consonância ao resultado encontrado na pesquisa (informação verbal) ${ }^{3}$.

Destaca-se ainda, que a visita domiciliar é instrumento ideal para a educação em saúde. É mediante as troca de informações durante as visitas, que se dá o contexto de vida de cada um e da família,

\footnotetext{
${ }^{3}$ Informação fornecida pela Secretaria Municipal de Saúde do município de Palmas, Palmas - TO, em agosto de 2014.
}

pois cada domicílio apresenta uma realidade e necessidade.

Neste contexto, percebe-se a importância do ACS na identificação das necessidades de saúde e na continuidade do cuidado, pois ele é o responsável por detectar as demandas da população e acompanhar a saúde das famílias, o que é preciso ser feito diariamente através da visita domiciliar (KEBIAN; ACIOLI, 2014).

Segundo o mesmo autor, a visita domiciliar é uma das principais atividades que permite ao ACS conhecer o contexto social $\mathrm{e}$ identificar as necessidades de saúde das famílias assistidas pela equipe, permitindo uma maior aproximação com os determinantes do processo saúde-doença.

A visita domiciliar na ESF é um instrumento utilizado pelas equipes para inserção e conhecimento do contexto de vida da população, assim como estabelecimento de vínculos entre profissionais e usuários. Ainda, visa atender as diferentes necessidades de saúde, preocupando-se com a infraestrutura (habitação, higiene, saneamento entre outros) existente nas comunidades e o atendimento à saúde das famílias ( MARIN et al., 2011).

A visita domiciliar deve ser realizada de forma sistematizada pelas equipes de saúde da família. Para isso, são traçadas formas de planejar, executar, registrar e avaliar a ação. O planejamento possibilita conhecer a história familiar previamente, favorecendo a aproximação e a orientação na visita domiciliar. Além de definir os dados que precisam ser levantados junto à família para serem agregados ao projeto terapêutico da família e ao plano de visita (ABRAHÃO; LAGRANGE, 2007).

A identificação de todas essas informações possibilitou caracterizar o perfil do ACS, reforçando o seu conceito de trabalhador sui generis, por ser um trabalhador único em seu gênero, de identidade comunitária, que por diversas vezes realiza atividades 
que extrapolam o campo da saúde, por reunir características tão singulares que o aproxima de sua comunidade, fortalecendo vínculos através do acolhimento, respeito e solidariedade.

O ACS é o profissional que conhece como nenhum outro os determinantes sociais da saúde na população que atende, visto que partilha destes mesmos determinantes, logo, o seu discurso tem grandes chances de ter aplicabilidade na realidade dos sujeitos (OLIVEIRA JÚNIOR, 2012).

\section{CONCLUSÃO}

Os resultados do estudo apontaram para algumas questões importantes sobre a identidade dos Agentes Comunitários de Saúde (ACS), principalmente no tocante à formação. $\mathrm{O}$ perfil que emerge no estudo é semelhante ao proposto pela Política Nacional de Atenção Básica, reafirmando a necessidade de inserir esses profissionais em processos de educação permanente, o que poderá contribuir na qualificação de suas práticas do ato de cuidar em saúde, junto aos indivíduos, famílias e comunidades, tendo em vista que essa necessidade vem sendo pautada pela realidade que ele enfrenta a partir da sua atuação. Estas questões relacionadas à formação do ACS impactam muito no seu reconhecimento como profissional de saúde e na construção de sua identidade profissional.

Nesse contexto, o resultado encontrado, ratifica que no município de Palmas, a educação permanente para os ACS, encontra-se incipiente. Fato este, que reforça a necessidade da implantação e/ou implementação de projetos formativos que venham auxiliar na compreensão dos momentos teóricopráticos, objetivando incentivar e provocar transformações nas orientações à comunidade, com incorporação de conhecimentos, atitudes e habilidades.

Todos os autores declararam não haver qualquer potencial conflito de interesses referente a este artigo.

\section{REFERÊNCIAS}

ABRAHÃO A.L; LAGRANGE V., A Visita Domiciliar como uma Estratégia da Assistência no Domicílio. In: Morosini MVGC, Corbo AMDA (org.). Modelos de atenção e a saúde da família. Rio de Janeiro: EPSJV/Fiocruz; 2007. p. 151-171.

ANDRADE, F.M.O. O Programa de Saúde da Família no Ceará. Fortaleza. Dissertação [Mestrado em Saúde Pública]. Universidade Federal do Ceará; 1998.

ÁVILA, M.M.M. O Programa de Agentes Comunitários de Saúde no Ceará: o caso de Uruburetama. Rio de Janeiro. Tese [Doutorado em Saúde Coletiva]. Universidade do Estado do Rio de Janeiro; 2006.

AZAMBUJA E.P et al. Significados do trabalho no processo de viver de trabalhadoras de um programa de saúde da família. Rev. Enf. 2007 Jan./Mar; vol.16 (1): $71-79$. )

BRASIL. Ministério da Saúde. Anais da reunião técnica dos pólos de capacitação, formação e educação permanente em saúde da família. Brasília, 2000 a.

Ministério da Saúde. Secretaria de Políticas de Saúde. Departamento de Atenção Básica. Guia Prático do Programa de Saúde da Família.Brasília: Ministério da Saúde; 2000 b.

Ministério da Saúde. Secretaria de Políticas de Saúde. Departamento de Atenção Básica. A implantação de Unidade de Saúde da Família : caderno 1. Brasília: MS, 2000 c.

Ministério da Saúde. Secretaria Executiva. Programa de Agentes Comunitários de Saúde (PACS). Brasília: Ministério da Saúde; 2001.

Lei n. 10.507, de 10 de junho de 2002. Cria a Profissão de Agente Comunitário de Saúde e dá outras providências; 2002. Disponível em: http://pfdc.pgr.mpf.mp.br/ . Acesso em 17de Setembro de 2013. 
.Ministério da Saúde. Referencial curricular para curso técnico de agente comunitário de saúde. Brasília, DF: Ministério da Saúde, 2004.

. Ministério da Saúde. Secretaria de Gestão do Trabalho e da Educação a Saúde. Departamento de Gestão da Educação na Saúde. A educação permanente entra na roda. Pólos de Educação Permanente em Saúde: conceitos e caminhos a percorrer. Brasília: Ministério da Saúde; 2005.

.Ministério da Saúde. Aprovada a Política Nacional de Atenção Básica, estabelecendo a revisão diretrizes e normas para organização da Atenção Básica para Programa Saúde da Família (PSF) e o Programa de Agentes Comunitários de Saúde (PACS). Portaria $\mathrm{n}^{\circ}$ 648, 28 de março de 2006.

http://www.brasilsus.com.br/legislacoes/gm/899648.html?q=] Acesso em 28 de maio de 2014.

Ministério da Saúde. Secretaria de Atenção à Saúde. Departamento de Atenção Básica. Guia prático do agente comunitário de saúde / Ministério da Saúde, Secretaria de Atenção à Saúde, Departamento de Atenção Básica. Brasília: Ministério da Saúde; 2009 a. 260 p.

Ministério da Saúde. Portaria n. ${ }^{\circ} 2.488$, de 21 de outubro de 2011. Aprova a Política Nacional de Atenção Básica, estabelecendo a revisão de diretrizes e normas para a organização da Atenção Básica, para a Estratégia de Saúde da Família (ESF) e o Programa de Agentes Comunitários de Saúde (PACS). Diário Oficial da União, 2011.2 Disponível: http://www.saude.mt.gov.br/atencao-asaude/arquivo/2581/portarias Acesso: 27 de Julho de 2014.

CARVAlHO, P.M.G., Práticas Educativas em Saúde: Ações dos Enfermeiros na estratégia Saúde da Família. 2009. 84 f. Dissertação (Mestrado em Enfermagem) , Universidade Federal do Piauí, Teresina - PI, 2009.

CHINELLI, F. et al. A Reconfiguração Gerencial do Estado Brasileiro e os Trabalhadores da Saúde nos anos 1990. In: Vieira $M$ et al (orgs). Para Além da Comunidade: Trabalho e Qualificação dos Agentes Comunitários de Saúde. Rio de Janeiro: EPSJV; 2011: 33 - 78

ELLIS, J.R.; HARTLEY, C.L. Enfermagem Contemporânea: desafios, questões e tendências. Tradução de Maria Virginia Godoy da Silva. $5^{\text {a }}$ ed. Porto Alegre: Artmed; 1998.
FERRAZ L.; AERTS DRGC. O Cotidiano de Trabalho do Agente Comunitário de Saúde no PSF em Porto Alegre.Ciências e Saúde Coletiva. 2005 Dez; vol. 10 (02): 347 - 355.

GOMES K.O., COTTA R.M.M., CHERCHIGLIA M.L, MILTRE S.M, BATISTA R.S. A práxis do agente comunitário de saúde no contexto do programa saúde da família: reflexões estratégicas. Saúde e Sociedade. 2009; 18 (4):744-55.

IBGE - Instituto Brasileiro de Geografia e Estatística. Censo 2010. Rio de Janeiro: IBGE; 2010. Disponível em: http://www.censo2010.ibge.gov.br. Acesso em 10 de Novembro de 2012.

IBGE, Nota Técnica - Estimativas da População dos Municípios Brasileiros com data de Referência em $1^{\circ}$. De Julho de 2014. Diretoria de Pesquisas -DPE ,Coordenação de População e Indicadores Sociais COPIS , Gerência de Estudos e Análises da Dinâmica Demográfica - GEADD. 2014.

INMET - INSTITUTO NACIONAL DE METEOROLOGIA. Brasília, INMET - Instituto Nacional de Meteorologia/Ministério da Agricultura Pecuária e Abastecimento, 2014. Disponível em: http://www.inmet.gov.br/portal/ Acesso em 10 de Agosto de 2014.

KEBIAN, L.V.A; ACIOLI , S. A visita domiciliar de enfermeiros e agentes comunitários de saúde da Estratégia Saúde da Família. Rev. Eletr. Enf. [Internet]. 2014jan/mar;16(1):161-9.Disponível: http://www.fen.ufg.br/fen_revista/v16/n1/pdf/v16n1a 19.pdf Acesso: 18 de Agosto de 2014.

LOBIONDO-WOOD, G.; HABER, J. Pesquisa em Enfermagem: métodos, avaliação crítica e utilização. $4^{\text {a }}$ edição. Rio Janeiro: Guanabara Koogan, 2001, 330 p.

LOPES, M.R et al. A Disputa sobre os Sentidos do Trabalho e da Formação dos Agentes Comunitários de Saúde. In: Vieira $\mathrm{M}$ et al (orgs). Para Além da Comunidade: Trabalho e Qualificação dos Agentes Comunitários de Saúde. Rio de Janeiro: EPSJV; 2011: 161 - 208.

LUNARDELO, S. R.O trabalho do agente comunitário de saúde nos Núcleos de Saúde da Família em Ribeirão Preto - São Paulo.2004. 156 f. Dissertação (Mestrado em Enfermagem em Saúde Pública) - Escola de Enfermagem de Ribeirão Preto, Universidade de São Paulo, Ribeirão Preto, 2004.

MARIN M.J. S, GOMES R, JUNIOR A.C. S, NUNES C.R. R, CARDOSO C.P, OTANI M.P et al. 
O sentido da visita domiciliária realizada por estudantes de medicina e enfermagem: um estudo qualitativo com usuários de unidades de saúde da família. Ciências e Saúde Coletiva. 2011; 16(11): 4357-65.

MARQUES, T.C; As atividades de agentes comunitários de saúde e a promoção do uso correto de medicamentos em unidades do Distrito de Saúde Oeste de Ribeirão Preto - SP, 105 f, Dissertação (Mestrado - Programa de Pós-Graduação em Enfermagem. Área de Concentração: Enfermagem Fundamental) - Escola de Enfermagem de Ribeirão Preto da Universidade de São Paulo.2008.

MALFITANO APS, LOPES RE. Educação popular, ações em saúde, demandas e intervenções sociais: o papel dos agentes comunitários de saúde. Caderno Cedes. 2009; 29(79): 361-72.

MARTINS C.L, OLIVEIRA L.S. S, RODRIGUES M.A, WATANABE H.A.W e JACOMO Y.A. Agentes comunitários nos serviços de saúde pública: elementos para uma discussão. Revista Saúde Debate. 1996 Jun. Vol. 51: 38-43.

MINAYO, M.C. S, D'elia J.C e SUITONE E. Programa de Agentes de Saúde do Ceará: estudo de caso. Fortaleza: Unicef; 1990.

MODESTO, Maria do Socorro Andrade; GRILLO, Luciane Peter; PROSPERO, Elisete NavasSanches and MARIATH, Aline Brandão. Avaliação de curso técnico de agente comunitário de saúde sob a ótica dos egressos. Trab. educ. saúde [online]. 2012, vol.10, n.3, pp. 387-406. ISSN 1981-7746.

MOTA, R.R.A ; DAVID, H.M.S.L. A Crescente Escolarização do Agente Comunitário de Saúde: uma indução do processo de trabalho?Trab. Educ. Saúde. 2010 Jul/Out; vol. 08 (02): 229 - 248.

NEGRI, L. S. A.; RUY, G. F.; COLLODETTI, J. B.;PINTO, L. F., SORANZ, D. R.Aplicação de um instrumento pra detecção precoce $e$ previsibilidade de agravos na população idosa.Ciência e Saúde Coletiva, v.9, n.4, p.1033-46, 2004.

OLIVEIRA JÚNIOR, J.G. Agentes Comunitários de Saúde: fatores restritivos e facilitadores do seu trabalho na Estratégia de Saúde da Família. 2012. 137f. Dissertação (Mestrado em Ciências na Área de Saúde Pública) - Escola Nacional de Saúde PúblicaSergio Arouca, FOICRUZ, Rio de JaneiroRJ, 2012.
ROSA, W. A. G.; LABATE, R. C. Programa Saúde da Família: a construção de um novo modelo de assistência. Revista Latino-am Enfermagem, v. 13, n. 6, p.1027-34, 2005.

SANTOS, L.P.G.S., A atuação do agente comunitário de saúde em São Bernardo do Campo: possibilidades e limites para a promoção da saúde. São Paulo. Dissertação [Mestrado em Enfermagem em Saúde Pública] Universidade de São Paulo; 2006.

SANTOS K.T., SALIBA N.A., MOIMAZ S.A.S., ARCIERI, R.M e CARVALHO, M.L. Agente Comunitário de Saúde: perfil adequado a realidade do Programa de Saúde da Família?Ciências \& Saúde Coletiva. 2011 Jul; vol. 16 (Supl. 1): 1023 - 1028).

SEOANE AF, FORTES PAC. A percepção do usuário do Programa Saúde da Família sobre a privacidade e a confidencialidade de suas informações. Saúde Soc. 2009; 18(1): 42-9.

SILVA, J.A ; DALMASO, A.S.W. Agente Comunitário de Saúde: o ser, o saber, o fazer. Rio de Janeiro: Fiocruz; 2002.

SOUSA, M.F., Agentes Comunitários de Saúde: Choque de Povo! São Paulo: Hucitec. 2001.

TEIXEIRA R.A; MISHIMA S.M. Perfil dos Trabalhadores de Enfermagem no PSF. Rev. Bras. de Enf. 2000 Jul/Set; vol. 53 (3): 386 - 400.

TOMAZ, J.B.C. O Agente Comunitário de Saúde não deve ser um "super-herói". Interf. - Comunicação, Saúde e Educação. 2002 Fev; vol. 6 (10): 75 - 94. 